Notas Clínicas

\title{
Vasculitis de pequeño vaso en un paciente con un adenocarcinoma de colon
}

\author{
M. J. Oruezábal Morenoํㅜ, M. R. Burón Fernández²
}

\section{Resumen}

Los pacientes oncológicos pueden desarrollar durante el seguimiento una vasculitis cuyo diagnóstico por su rareza es difícil aunque de vital importancia dada su alta mortalidad sin tratamiento y con el fin de evitar errores en el estadiaje al interpretar las manifestaciones clínicas como secundarias al tumor.

Se presenta un caso de una vasculitis de pequeño vaso P-ANCA positivo, con afectación pulmonar aislada, en un paciente con un adenocarcinoma de colon. Este caso demuestra la importancia en reconocer la enigmática y variable presentación clínica de las vasculitis sistémicas, un síndrome tan infrecuente como desconocido.

\section{Palabras clave:}

Vasculis. P-ANCA. Hemorragia alveolar difusa. Adenocarcinoma de colon.

Oncología, 2005; 28 (6):297-303

\footnotetext{
${ }^{1}$ Sección Oncología Médica

2 Servicio de Medicina Interna

Hospital General La Mancha Centro

Alcázar de San Juan (Ciudad Real)
} 


\section{Summary}

Oncologic patients can develop a vasculitis during the follow-up, whose diagnosis is difficult due to the rarity of the process. Notwithstanding it is of vital importance because the high mortality rate when it is not treated, and also because its diagnosis avoids staging mistakes when interpreting the clinical manifestations secondary to the tumor.

We report a case of p-ANCA-positive small vessel vasculitis associated to an isolated lung hemorrhage in a patient with colon adenocarcinoma, demonstrating the importance of recongnizing the enigmatic and variable clinical presentation of the systemic vasculitis, an infrequent and badly known syndrome.

Key words: Vasculitis. P-ANCA. Diffuse alveolar hemorrhage. Colon adenocarcinoma.

\section{Introducción}

La vasculitis es un síndrome infrecuente que pueden aparecer en neoplasias hematológicas y no hematológicas en ausencia de metástasis en los órganos afectados.

Son escasos los casos publicados en la literatura por lo que su patogenia es desconocida siendo el diagnóstico y tratamiento un tema de controversia con implicación de múltiples especialidades.

La vasculitis de vasos pequeños es la más frecuente afectando a vénulas, capilares y arteriolas como consecuencia de un proceso inflamatorio que suele estar mediado por complejos inmunes. La afectación pulmonar aislada es rara aunque recientemente se ha descrito una vasculitis de pequeño vaso conocida como capilaritis pulmonar pauci-inmune, asociada a anticuerpos perinucleares antineutrófilos (p-ANCA) y causante de hemorragia alveolar difusa cuyo diagnóstico requiere un alto índice de sospecha al no existir lesiones endobronquiales y ser los hallazgos histopatológicos inespecíficos. El descubrimiento de los anticuerpos ANCA asociados a las vasculitis de pequeño vaso ha facilitado su reconocimiento y el mecanismo fisiopatológico implicado.

En este artículo se presenta el caso de un varón de 72 años con una hemorragia pulmonar difusa asociada a una vasculitis p-ANCA positiva diagnosticada tras ser intervenido de un adenocarcinoma de colon.

\section{Caso clínico}

Varón de 72 años, diagnosticado de diabetes mellitus tipo 2 en tratamiento con glizipida desde hacía
5 años y un adenocarcinoma de colon estadio C2 Astler-Coller en marzo de 2003, intervenido con hemicolectomía derecha y quimioterapia adyuvante con 5-FU-LV según esquema Clínica Mayo.

Ingresó en noviembre de 2003 con un cuadro de síndrome constitucional y esputos hemoptoicos persistentes desde hacía un mes aunque en cantidad escasa. En la exploración física destacaba palidez mucocutánea y crepitantes secos en ambas bases pulmonares.

En la analítica tenía anemia ferropénica ( $\mathrm{Hb} 6,1$ g/dL, MCV 73, HCM 21, sideremia 15, ferritina 3, transferrina 285), aumento de VSG (89 mm), fibrinógeno y PCR, e hipergammaglobulinemia policlonal. Anticuerpos ANA y anti-DNA negativos. El estudio de bacilos ácido-alcohol resistentes (BAAR)

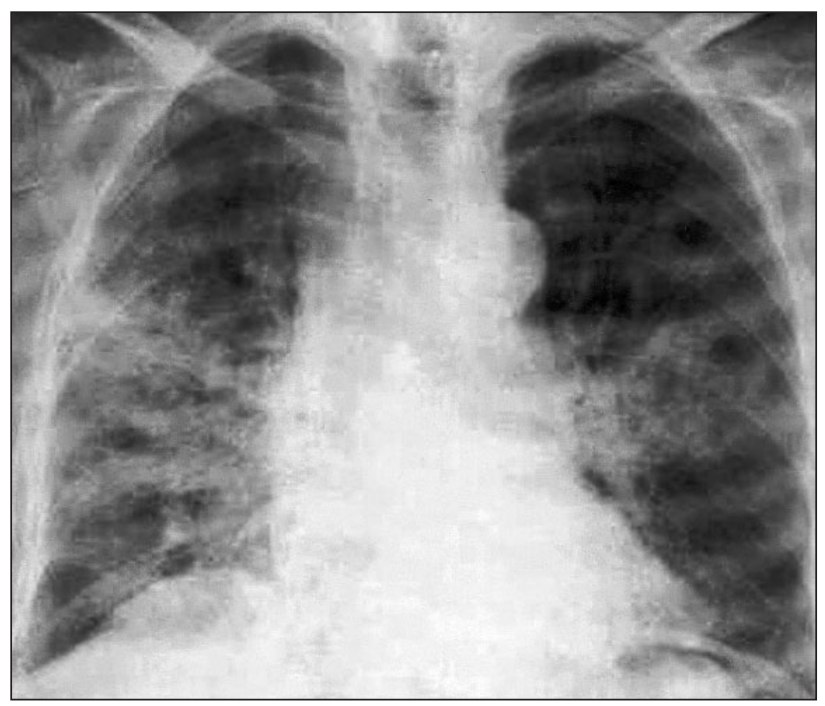

Figura 1. Radiografía de tórax. Patrón alveólo intersticial de predominio en ambas bases. 
en esputo fue negativo. Los marcadores tumorales mostraban CA19.9 de $8 \mathrm{U} / \mathrm{mi}(<37 \mathrm{U} / \mathrm{ml})$ y CEA de $3 \mathrm{U} / \mathrm{mi}(<5 \mathrm{U} / \mathrm{ml})$.

En las pruebas de imagen se objetivó en la radiografía de tórax y en el TAC toracoabdominal (Fig. 1) un patrón alvéolo-intersticial de predominio en ambas bases. La broncoscopia no mostró ninguna lesión endobronquial ni signos de sangrado .

Se realizó una ecografía abdominal, gastroscopia, colonoscopia y fibrolaringoscopia directa que fueron normales.

Fue dado de alta con hierro oral y seguimiento ambulatorio por la sospecha de un proceso tumoral no filiado tanto por la clínica de ingreso como por los resultados analíticos.

Reingresó en febrero de 2004 por insuficiencia respiratoria aguda $(\mathrm{pH} \mathrm{7,36;} \mathrm{pCO2} 44 \mathrm{mmHg} ; \mathrm{pO} 2$ 56 mmHg; saturación O2 66,6\%) que precisó oxigenoterapia a flujos altos. En la radiografía de tórax destacaba un patrón alveolointersticial bilateral, con afectación de ambas bases y campo medio derecho siendo diagnosticado de una neumonia bilateral y recibió tratamiento antibiótico con ceftriaxona y eritromicina. En la analítica destacaba una leucocitosis con desviación izquierda, anemia microcítica e hipocrómica, aumento de VSG y de LDH. El análisis de orina elemental fue normal. Los hemocultivos fueron repetidamente negativos; BAAR en orina $\mathrm{y}$ esputo negativo; serología VHC, VHB, CMV, VIH, Legionella, Fiebre Q, Mycoplasma y Clamydias negativos. La enzima convertidora de angiotensina estaba dentro del rango de la normalidad. Los marcadores tumorales fueron normales (CEA $3 \mathrm{U} / \mathrm{ml}$ y CA19.9 20 U/ml). La TAC toracoabdominal mostró a nivel pulmonar un patrón reticular intersticial difuso con un engrosamiento interlobulillar, más marcado en los segmentos posteriores y un patrón en vidrio delustrado en ambas bases. Se realizó una broncoscopia donde no se observaron lesiones endoluminales en el árbol bronquial y bronquios segmentarios, con citología negativa (Fig. 2). La biopsia transbronquial fue no representativa. Dada la situación clínica del enfermo se realizó un Lavado Bronquialveolar que mostró un infiltrado neutrofílico con abundantes macrófagos con hemosiderina. El cultivo y citología fueron negativos para gérmenes y células tumorales.

El paciente seguía sin mejoría clínica ni radiológica, y ante la sospecha, por los hallazgos en el la-

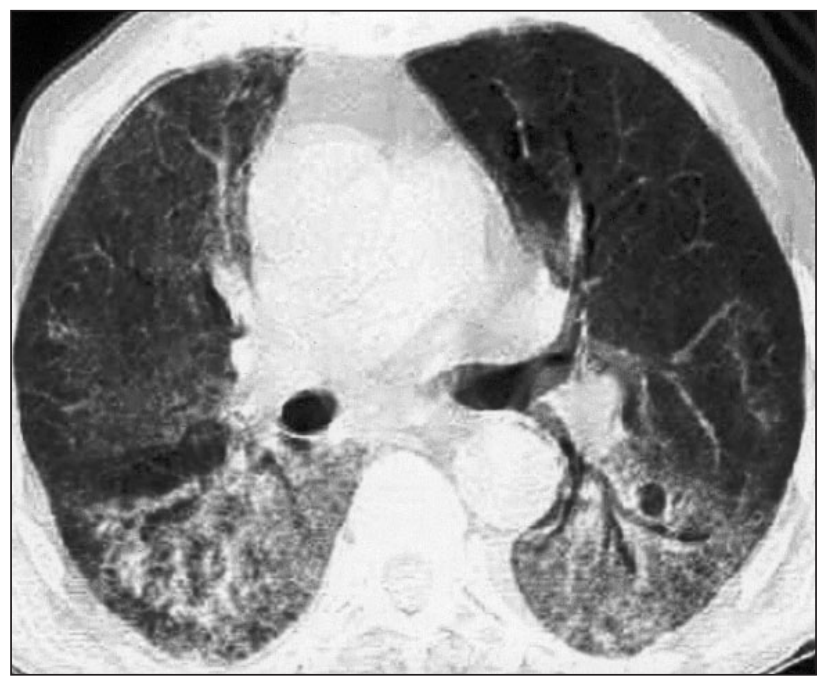

Figura 2. TAC de tórax: patron reticular difuso con engrosamiento interlobulillar, más marcado en segmentos posteriores y un patrón en vidrio delustrado en ambas bases.

vado bronquialveolar, de una hemorragia pulmonar difusa se solicitó un estudio inmunológico que mostró titulos 1/650 para los anticuerpos anticitoplasma de neutrófilo (ANCA) perinucleares, los anticuerpos antinucleares (ANA), anti-ADN, antiRO y anti-La, anticuerpos antimembrana basal, anticoagulante lúpico y factor reumatoide fueron negativos.

Se inició tratamiento con metilprednisolona (dosis iniciales de $1 \mathrm{mg} / \mathrm{kg}$ peso/día durante 20 días, con reducción progresiva en las siguientes semanas).

A partir del inicio del tratamiento esteroide el enfermo evolucionó favorablemente, tanto clínicamente como por gasometría. Radiológicamente, se mantuvo la presencia de infiltrados pulmonares bilaterales, apreciándose en el último control efectuado un patrón de fibrosis pulmonar difusa.

A los 3 meses del alta, el paciente no requería oxigenoterapia y se mantenía con prednisona oral en pauta descendente.

Posteriormente, el paciente fue revisado de forma periódica en la consulta externa del hospital. En la actualidad, tras un año de evolución, se encuentra en una buena situación clínica, sin anemia (Hb 13 $\mathrm{g} / \mathrm{dL}$ ), normalización de la VSG, reactantes de fase aguda y negativización de los anticuerpos p-ANCA. Se ha vuelto a realizar una colonoscopia, TAC toracoabdominal y marcadores tumorales sin apreciar en el momento actual recidiva tumoral. 


\section{Discusión}

La asociación entre vasculitis y cáncer ha sido ampliamente documentada en las últimos años, encontrando neoplasias malignas en el 4,5-8\% de los pacientes con vasculitis, principalmente hematológicas, aunque también tumores sólidos ${ }^{1}$. Además, los pacientes oncológicos pueden desarrollar durante la evolución vasculitis asociada al tumor o inducida por fármacos. En la literatura se han descrito vasculitis asociada principalmente a neoplasias linfoproliferativas aunque también con leucemias, mielomas y tumores no hematológicos como cáncer de riñón, nasofaringe, pulmón, próstata, mama y colon $^{2-6}$. En otros casos se ha relacionado con el fármaco administrado como gemcitabina, 5-fluorouracilo, tamoxifeno y después del transplante autólogo de médula ósea, mejorando tras la suspensión del citostático y la administración de metilprednisolona y/o ciclofosfamida ${ }^{7-9}$. En una estudio retrospectivo realizado Cleveland Clinic Foundation en un periodo de 18,5 años hubo 12 vasculitis asociada a tumor que supuso $0,5 \%$ del total de las vasculitis diagnosticadas, siendo la vasculitis leucocitoclástica cutánea la más frecuente, seguida por arteritis de células gigantes, panarteritis nodosa y enfermedad de Wegener $^{10}$.

El presente caso clínico muestra una vasculitis ANCA positivo que compromete vasos sanguíneos pequeños durante el seguimiento de un paciente con cáncer de colon.

Las vasculitis son enfermedades producidas por inflamación de la pared de los vasos sanguíneos de distintas causas, que pueden presentarse con múltiples cuadros clínicos según el tamaño y localización de los vasos afectados.

Las vasculitis paraneoplásicas se definen como aquellas vasculitis asociados al cáncer que ocurren a distancia de la neoplasia primaria e inducidas por antígenos tumorales que desencadenan una reacción inmune. Pueden preceder al diagnóstico de la neoplasia; ser marcadores de recidiva tumoral o aparecer concomitantemente o después del diagnóstico del tumor. El curso clínico de las vasculitis paraneoplásica es habitualmente paralelo al del tumor. De este modo, la curación de la neoplasia usualmente, aunque no invariablemente, resulta en la regresión de la vasculitis, sin embargo cuando la vasculitis no es simultánea al tumor el diagnóstico es difícil pu- diendo ser la evolución independiente y requiriendo un tiempo de seguimiento prolongado para considerar la vasculitis como paraneoplásica ${ }^{11,12}$.

Las vasculitis necrotizante de pequeño vaso es la forma de presentación más frecuente de vasculitis del adulto asociada a neoplasias, y se caracterizan por comprometer vasos pequeños como arteriolas, capilares y vénulas, compartir la asociación a ANCA y una gran cantidad de manifestaciones clínicas, incluyendo el compromiso renal que consiste en una glomerulonefritis focal con escasos depósitos inmunes o ausencia de ellos. Incluyen cuadros como la poliangeítis microscópica, la granulomatosis de Wegener, el Síndrome de Churg-Strauss, la vasculitis crioglobulinémica y la Púrpura de Schönlein-Henoch $^{13}$. La capilaritis pulmonar pauci-inmune aislada ha sido descrita recientemente como una vasculitis de pequeño vaso que afecta unicamente al pulmón, de características y pronóstico similar a la poliangeitis microscópica aunque sin afectación renal $^{14}$.

La etiología de los síndromes vasculíticos es desconocida. Se acepta que el depósito de inmunocomplejos de tamaño mayor de 19 S, con la activación del complemento y la consiguiente inflamación vascular, es el mecanismo inmunopatológico fundamental. Sin embargo, no siempre es posible demostrar la presencia de los inmunocomplejos y sólo en muy pocos casos se puede establecer su etiología por la demostración del antígeno responsable, como ocurre en las vasculitis asociadas al virus de la hepatitis B y en determinados casos de vasculitis asociada a neoplasias, en las que antígenos de naturaleza tumoral actuarían como desencadenantes. Se ha concedido importancia en la fisiopatogenia de las vasculitis a otros anticuerpos dirigidos contra constituyentes enzimáticos (proteinasa 3, elastasa, mieloperoxidasa, etc) del citoplasma de los neutrófilos (ANCA). De ellos, los p-ANCA con especificidad antimieloperoxidasa se relacionan con la variedad microscópica de la PAN y los c-ANCA dirigidos frente a la proteasa 3 con la enfermedad de Wegener ${ }^{15}$.

Los pacientes pueden consultar por síntomas constitucionales tales como malestar general, astenia, pérdida de peso y fiebre. La hemorragia pulmonar se observa en el 12-29\% de los casos y es un factor que contribuye a la morbilidad y mortalidad de estos pacientes. La hemoptisis suele variar desde 
un esputo hemoptoico hasta una franca hemorragia, que puede evolucionar de días a semanas. La hemoptisis se asocia a disnea y anemia ferropénica y progresa rápidamente a una insuficiencia respiratoria y a una hipoxemia severa. En algunos casos, la vasculitis de pequeños vasos puede producir un proceso segmentario e intersticial evolucionando a una fibrosis cuando existe un daño alveolar previo $^{16}$.

La presentación radiológica aguda se caracteriza por la presencia de infiltrados de ocupación alveolar de predominio perihiliar y bilateral que van confluyendo para configurar una imagen de completa consolidación del espacio aéreo, respetando generalmente los ápices y la periferia de los pulmones. Estos infiltrados se resuelven en una a dos semanas, pero con los episodios repetidos de sangrado se puede desarrollar fibrosis intersticial ${ }^{17}$.

La broncoscopia con Lavado Broncoalveolar puede ser de utilidad en el diagnóstico de hemorragia pulmonar oculta, sobre todo cuando el cuadro clínico simula otras formas de enfermedad intersticial, pudiendo diagnosticarse con seguridad al encontrar macrófagos alveolares cargados con hemosiderina, aunque carece de sensibilidad para establecer la etiología de la hemorragia pulmonar.

El papel de la biopsia pulmonar es controvertido ya que la mayoría de las veces los hallazgos histopatológicos son inespecíficos, además la sensibilidad de la biopsia por fibrobroncoscopia es baja, entorno al 7\%, ni el estado general del paciente permite realizar en la fase aguda una biopsia por toracoscopia o abierta $^{18}$.

Desde el punto de vista anatomopatológico, con frecuencia la vasculitis de pequeño vaso pulmonar se caracteriza por la presencia de una capilaritis pulmonar necrotizante, en ausencia de evidencia histológica de arteriolitis o venulitis, con acumulación de un intenso infiltrado intersticial neutrofílico, frecuente cuerpos picnóticos y edema abundante. La presencia de sangre en los espacios alveolares distales dificulta todavía más el diagnóstico por lo que serán las pruebas serológicas las que apoyen la sospecha diagnóstica ${ }^{19}$.

La determinación de anticuerpos anticitoplasma de neutrófilos (ANCAS), ha revolucionado el diagnóstico en la vasculitis. Se distinguen dos tipos de ANCAS: un patrón citoplasmático que representa anticuerpos frente a la proteinasa 3 de los neutrófi- los, los cuales son altamente sensibles y específicos para Granulomatosis de Wegener, y un segundo patrón, el perinuclear que representa anticuerpos contra la mieloperoxidasa de los neutrófilos, que se ha relacionado con poliangeítis microscópica, síndrome pulmón riñón idiopático, síndrome de ChurgStrauss, LES inducido por hidralazina y sarcoidosis.

Dado que no se conoce con claridad si estos anticuerpos desempeñan algún papel patogénico y el amplio espectro de patologías en las que pueden hallarse, su interpretación debe realizarse en conjunto con el cuadro clínico del paciente. A pesar de la limitaciones de la prueba, hay estudios que sugieren que en situaciones especiales, en las que el paciente tenga una alta probabilidad de tener una enfermedad asociada a ANCAs, estos pueden sustituir la confirmación histopatológica, siendo esto particularmente cierto en los casos de hemorragia alveolar secundaria capilaritis pulmonar pauci-inmune, en los cuales la mortalidad es alta y los hallazgos de la biopsia son frecuentemente inespecíficos ${ }^{20}$.

El reconocimiento de la vasculitis es clínicamente importante, ya que pueden complicar el estadiaje del cáncer simulando enfermedad metastásica; por otra parte las verdaderas metástasis pueden comportarse como vasculitis y ser ofertada erróneamente una cirugía radical.

El diagnóstico pasa por descartar otras causas de hemorragia alveolar difusa con anticuerpos ANCA positivos como el síndrome de Goodpasture y LES.

La determinación serológica de marcadores para el diagnóstico de LES confirmarán el diagnóstico: los ANAS están presentes en el 95\% de los pacientes con enfermedad activa, usualmente a títulos altos; y los anticuerpos anti DNA de doble cadena están presentes en $50-75 \%$ de los pacientes siendo más específicos para el diagnóstico de LES $^{21}$.

Igualmente, el diagnóstico de Síndrome de Goodpasture se facilita con los estudios de inmunoflorescencia en tejidos y determinación sérica para anticuerpos antimembrana basal glomerular (antiMBG). La demostración de depósitos lineares de inmunoglobulinas a lo largo de la membrana basal alveolar o glomerular son el patrón más específico para el diagnóstico de síndromes pulmón-riñón secundarios a Síndrome de Goodpasture ${ }^{22}$.

En nuestro caso se estableció el diagnóstico de vasculitis de pequeño vaso asociada al tumor según la clasificación de Chapel-Hill por la presencia a tí- 
tulos altos de anticuerpos p-ANCA, la ausencia de anticuerpos anti-MBG, el antecedente oncológico, y la respuesta a los esteroides, ya que como era de esperar los hallazgos histopatológicos fueron inespecíficos. La denominación de vasculitis paraneoplásica es compleja, como ocurre en la mayoría de los síndromes paraneoplásicos, dado que la vasculitis no apareció simultánea al diagnóstico del cáncer de colon y no existen marcadores específicos que confirmen el diagnóstico, aunque la baja frecuencia de capilaritis pulmonar pauci-inmune en la población general y su aparición en un periodo de tiempo próximo al diagnóstico de cáncer de colon apoyan que ambas enfermedades pueden estar asociadas, tal como se ha señalado en otros casos similares referidos en la literatura ${ }^{23}$.

La hemorragia alveolar difusa es una urgencia médica, cuyo tratamiento se debe iniciar antes de determinar el diagnóstico específico. El tratamiento fundamentalmente con esteroides $(0.5-1 \mathrm{mg} / \mathrm{kg}$ con disminución progresiva en 3-6 meses) ha mejorado drásticamente el curso y el pronóstico de la vasculitis. La remisión clínica alcanza hasta el $70 \%$ de los casos tratados, aunque un $25 \%$ presentaran recidivas, generalmente asociadas a una disminución en la dosis de corticoides y acompañada de un aumento de los anticuerpos p-ANCA.

En los casos refractarios a los corticoides está indicado el uso de corticoides a altas dosis $(1.5 \mathrm{mg} / \mathrm{kg}$ durante largos periodos) y la asociación de inmunosupresores como ciclofosfamida, azatioprina e Inmunoglobulina endovenosa ${ }^{24}$. Los pacientes con hemorragia pulmonar pueden beneficiarse de la plasmaféresis ${ }^{25}$. Se ha descrito el uso de micofenolato de mofetilo y esteroides orales para el tratamiento a largo plazo con menor cantidad de efectos colaterales y con porcentajes de remisión y recaída similares a los reportados con otros esquemas ${ }^{26}$.

La valoración de la naturaleza paraneoplásica de la vasculitis constituye un desafío médico, ya que las manifestaciones clínicas de la vasculitis pueden ser indistinguibles de las producidas por el tumor y el diagnóstico de la naturaleza paraneoplásica sólo es posible tras descartar otras enfermedades cuya clínica, radiología y histopatología son similares, sin embargo es indiscutible la relación de la vasculitis de pequeño vaso y el cáncer cuyo conocimiento es transcendental para un adecuado abordaje terapeútico de ambas entidades.

\section{Bibliografía}

1. Pankhurst T, Savage CO, Gordon C, Harper L. Malignancy is increased in ANCA-associated vasculitis. Rheumatology 2004;43(12):1532-1535.

2. Sanchez NB, Canedo IF, García-Patos PE, de Unamuno Perez P, Ben AV, Pascual AM. Paraneoplastic vasculitis associated with multiple myeloma. J Eur Acad Dermatol Venereol 2004; 18(6):731-735.

3. T Ponge, D Boutoille, A Moreau, P Germaud, G Dabouis, $\mathrm{T}$ Baranger, and $\mathrm{J}$ Barrier. Systemic vasculitis in a patient with small-cell neuroendocrine bronchial cancer Eur Respir J 1998; 12: 1228-1229.

4. Curgunlu A, Karter Y, Uyanik O, Tunckale A, Curgulu S. Leukocytoclastic vasculitis and renal cell carcinoma. Intern Med 2004; 43(3):256-257

5. Wang CC, Chen MJ, Ho HC, Hong HS. Urticarial vasculitis and dermatomyositis in a patient with nasopharingeal carcinoma. Cutis 2003; 72(5):399-402.

6. Hellmich A. Urinary bladder cancer in Wegener's granulomatosis: is it more than cyclophosphamide? Ann Rheum Dis 2004;63:1183-1185.

7. Birlik M, Akar S, Tuzel E, Onen E, Manisali M, Kirkali Z. Gemcitabine-induced vasculitis in advanced transitional cell carcinoma of the bladder. Cancer Res Clin Oncol 2004;130(2):122-125.

8. Drago F, Arditi M, Rebora A. Tamoxifen and purpuric vasculitis. Ann Intern Med 1990; 12: 965-966.

9. Seiden MV, O’Donnell WJ, Weinblatt M, Licht J. Vasculitis with recurrent pulmonary hemorrhage in a long-term survivor after autologous bone marrow transplantation. Bone Marrow Transplant 1990; 6: 345-347.

10. Hutson TE, Hoffman GS. Temporal concurrence of vasculitis and cancer: a report of 12 cases. Arthritis Care Res 2000;13(6):417-23.

11. Norris JH, Leeds J, Jeffrey RF. P-ANCA positive renal vasculitis in association with renal cell carcinoma and prolonged hydralazine therapy. Ren Fail 2003; 25(2):311-314.

12. Naschitz JE, Kovaleva J, Shaviv N, Rennert G, Yeshurun D. Vascular disorders preceding diagnosis of cancer: distinguishing the causal relationship based on Bradford-Hill guidelines. Angiology 2003;54(1):11-17.

13. Wilhelm S, Wolfgang G. Vasculitis in the seriously ill patient: Diagnostic approaches and terapeutic options in ANCA-associated vasculitis. Kidney Int 1998; 53: 39-44.

14. Green RJ, Ruoss SJ, Kraft SA, et al. Pulmonary capillaritis and alveolar hemorrhage: update in diagnosis and management. Chest 1996;110:1305-1316

15. Watz H, Hammerl P, Matter C, Degen M, Heimann F, Heitmann $\mathrm{H}$, et al. Bronchioloalveolar carcinoma of the lung associated with a highly positive p-ANCA-titer and clinical signs of microscopic polyangiitis. Pneumologie $2004 ; 58(7): 493-8$.

16. Jennette JC, Falk RJ. Small vessel Vasculitis. N Engl. J Med 1997; 337: 1512-1523. 
17. Travis WD, Colby TV. A clinic-pathologic study of 34 cases of diffuse pulmonary hemorrhage with lung biopsy confirmation. Am J Surg Pathol 1999; 14(12): 1112 1125 .

18. González-Gay MA, García-Porrúa C. Systemic vasculitis in adults in northwestern Spain, 1988-1997: Clinical and epidemiologic aspects. Medicine (Baltimore) 1999; 78: 292-308.

19. Marvin I Schwarz, Kevin K Brown. Small vessel vasculitis of the lung. Thorax 2000;55:502-510

20. Nolle B, Speck's V, Ludemann. ANCAS autoanticuerpos: their inmunodiagnostic valvue in Wegener granulomatosis. Ann Intern med 1989; 111: 28-40.

21. Murin S, Mathay R. Pulmonary Manifestations of LES Clin in Chest Medicine 1998; 19: 641-65.

22. Hudson B, Tryggvason K. Alport's Syndrome, Goodpastu- re's Syndrome and Tipe collagen. N Engl J Med 2003; 348:2543-2556.

23. Khachemoune A, Longo MI, Phillips TJ. Nodular vasculitis as a paraneoplastic presentation? Int J Dermatol. 2003;42(8):639-42.

24. Valente RM, Hall S. Vasculitis related disorders. In Kelly WN, Ruddy S. Textbook of Rheumatology. London, Saunders, 1997;1099.

25. Nachman PH. Treatment response and relapse in antineutrophil cytoplasmic autoantibody-associated microscopic polyangiitis and glomerulonephritis. J Am Soc Nephrol 1996; 7(1): 33-9.

26. Nowack R. Mycophenolate mofetil for maintenance therapy of Wegener's granulomatosis and microscopic polyangiitis: a pilot study in 11 patients with renal involvement. J Am Soc Nephrol 1999; 10(9): 1965-71.
Correspondencia:

Dr. M. J. Oruezábal Moreno

Hospital General La Mancha Centro

C/ Constitución, s/n

E-13600 Alcázar de San Juan (Ciudad Real)

E-mail: mauroo@sescam.jccm.es 